\title{
Formation of bridged bicycloalkenes via ring closing metathesis
}

\author{
Andrew Morehead, Jr. and Robert Grubbs* \\ The Arnold and Mabel Beckman Laboratory of Chemical Synthesis, Division of Chemistry and Chemical Engineering, California \\ Institute of Technology, Pasadena, California 91125, USA
}

Ring closing metathesis may be used in the formation of small ring bicycloalkenes from monocyclic diene precursors.

Ring closing metathesis (RCM), catalyzed by transition metal carbenes, has recently become an established method for the conversion of dienes to cyclic olefins. ${ }^{1}$ While many applications of RCM have been reported, ${ }^{2}$ only a few have involved the formation of bridged bicyclic compounds. The reported examples all involved macrocyclization (ring sizes $>12$ atoms) except one, which involved the formation of a seven-membered ring leading to a bicyclo[9.5.1] alkene ring system. ${ }^{3}$ Since small ring bridged bicycloalkanes and heterocycles are ubiquitous in natural products, the application of RCM in ring closures of this type would potentially lead to greatly broadened utility of this method.

A series of monocyclic diene substrates was synthesized $\dagger$ and the ring closure promoted by the well-defined ruthenium benzylidene complex ${ }^{4}$ was examined [eqn. (1)]. $\ddagger$ The results of

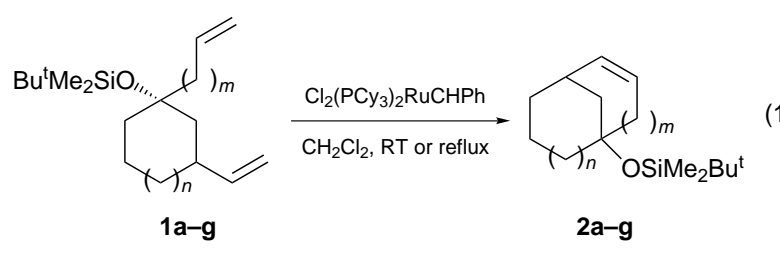

the cyclization reactions are summarized in Table 1 .

The ring closure of five-, six- and seven-membered rings to form [3.x.1], [4.x.1] and [5.x.1] bicycloalkenes is a relatively facile process (entries 1, 2, 5, 6 and 8), while ring closure of eight-membered rings to give bicyclo[5.x.1]alkenes does not occur (entries 3, 9 and 10). Variation of the reaction temperature, concentration of the diene and catalyst type (Mo catalyst $^{5}$ ) had no influence on the formation of the eightmembered rings, as starting material and oligomers were the only products isolated.

Formation of the bicyclo-[3.2.1]- and -[3.3.1]-alkenes when the six-membered ring is being closed is extremely facile (entries 1 and 6). In general, as the ring closure becomes slower, other processes such as dimerization may start to compete. Lower concentration disfavors the intermolecular coupling relative to the intramolecular ring closure, and allows clean formation of the products. Raising the temperature to that of refluxing $\mathrm{CH}_{2} \mathrm{Cl}_{2}$ was necessary only when the substrate was sterically hindered (entries 4 and 5) or the cyclization was very slow (entry 8).

The enthalpies of ring closure (including formation of ethylene) were calculated using the MM2 forcefield and are included in Table 1.6 In general, if the calculated enthalpy is less than $c a .10 \mathrm{kcal} \mathrm{mol}^{-1}$, the ring closure should be favorable due to the entropy gain from the generation of ethylene. An interesting comparision may be made between entry 5 and entry 3 , both of which are calculated to have $\Delta H \approx 13 \mathrm{kcal} \mathrm{mol}^{-1}$. For substrate 1 (entry 5), the kinetic favorability of ring closure for five-membered rings, coupled with the loss of ethylene to the atmosphere, drives the reaction to completion. Note that at higher concentrations (entry 4), the yield is much lower, as a result of a significant amount of polymer being formed, suggesting that endothermic ring closures are possible if the concentration is kept below the critical concentration for polymerization. In the case of the eight-membered ring (entry 3 ), although the same loss of ethylene would occur, the ring closure is sufficently slow as to be non-competitive with polymerization. Linderman et al. have recently reported the use of removable $\mathrm{Bu}_{3} \mathrm{Sn}_{\mathrm{Sn}}$ groups to overcome the entropic barrier allowing formation of eight-membered rings, ${ }^{7}$ a strategy that could potentially be applied here. The molecular mechanics also suggest that the silyl-protected alcohol plays a small role in the cyclization by decreasing the enthalpy of the ring flip to the bisaxial conformer needed for the ring closure by $c a$. $1 \mathrm{kcal} \mathrm{mol}^{-1}$ relative to the unprotected alcohol.

In conclusion, a number of small ring bridged bicycloalkenes have been synthesized from monocyclic dienes, demonstrating the power of ring closing metathesis to form carbocycles that contain a significant amount of strain.

A. T. M. acknowledges a postdoctoral fellowship from the National Institutes of Health (USA). We also thank Professor Dennis Dougherty for the use of the SGI Power Impact workstation and the software packages used for the calculations referred to in this paper.

Table 1 Conversion and ethalpies for ring closure of monocyclic dienes

\begin{tabular}{|c|c|c|c|c|c|c|c|c|}
\hline Entry & Substrate & $n$ & $m$ & Conc./M & {$[\mathrm{Ru}](\mathrm{mol} \%)$} & $T /{ }^{\circ} \mathrm{C}$ & Yield (\%) & $\begin{array}{l}\Delta H^{a /} \\
\mathrm{kcal} \mathrm{mol}^{-1}\end{array}$ \\
\hline 1 & 1a & 0 & 1 & 0.050 & 5 & RT & 87 & 1.2 \\
\hline 2 & $1 b$ & 0 & 2 & 0.005 & 5 & $\mathrm{RT}$ & $(>95 \text { Conv. })^{b}$ & 6.9 \\
\hline 3 & 1c & 0 & 3 & 0.005 & 10 & Reflux & Oligo & 13.1 \\
\hline 4 & 1d & 1 & 0 & 0.050 & 5 & Reflux & 41 & 13.3 \\
\hline 5 & 1d & 1 & 0 & 0.005 & 10 & Reflux & 84 & 13.3 \\
\hline 6 & $1 \mathrm{e}$ & 1 & 1 & 0.050 & 5 & RT & 95 & 1.7 \\
\hline 7 & 1f & 1 & 2 & 0.050 & 5 & $\mathrm{RT}$ & Trace & 10.0 \\
\hline 8 & 1f & 1 & 2 & 0.005 & 10 & Reflux & 96 & 10.0 \\
\hline 9 & $1 \mathrm{~g}$ & 1 & 3 & 0.050 & 5 & $\mathrm{RT}$ & Oligo & 17.5 \\
\hline 10 & 19 & 1 & 3 & 0.005 & 10 & Reflux & Oligo & 17.5 \\
\hline
\end{tabular}

${ }^{a}$ MM2 Calculations. ${ }^{b}$ Conversion by NMR spectroscopy. 


\section{Footnotes and References}

* E-mail: rhg@cco.caltech.edu

$\dagger$ The substrates were synthesized by addition of the appropriate Grignard reagent to 3-vinylcyclohexanone or cyclopentanone, and protection of the tertiary alcohol with the tert-butyldimethylsilyl group. The cis and trans isomers could be separated for the six- membered ring substrates, but not the five-membered ring substrates. The five-membered ring substrates were cyclized as mixtures and the product separated from the uncyclized trans diene by chromatography.

* Typical experiment procedure for 1-(tert-butyldimethylsilyloxy)bicyclo[3.3.1] non-6-ene (2e). The silyl ether protected diene 1 e $(630 \mathrm{mg}$, $2.25 \mathrm{mmol})$ was dissolved in $45 \mathrm{ml} \mathrm{CH} \mathrm{Cl}_{2}$ and treated with $\left[\mathrm{Cl}_{2}(\mathrm{Cy}\right.$ $\left.{ }_{3} \mathrm{P}\right)_{2} \mathrm{RuCHPh}$ (ref. 5) $92.4 \mathrm{mg}, 0.11 \mathrm{mmol}, 0.05$ equiv.]. The initial red solution rapidly turned light brown, was stirred for $4 \mathrm{~h}$, and the solvent was removed. The brown oil was purified by filtration through silica gel using hexane as the eluent to give a clear oil $(540 \mathrm{mg}, 2.14 \mathrm{mmol}, 95 \%): \delta_{\mathrm{H}}(300$ $\left.\mathrm{MHz}, \mathrm{CDCl}_{3}\right) 0.08(\mathrm{~s}, 6 \mathrm{H}), 0.86(\mathrm{~s}, 9 \mathrm{H}), 1.34-1.38(\mathrm{~m}, 2 \mathrm{H}), 1.45-1.86(\mathrm{~m}$, $6 \mathrm{H}), 2.13-2.32(\mathrm{~m}, 2 \mathrm{H}), 2.56-2.60(\mathrm{~m}, 1 \mathrm{H}), 5.52-5.57(\mathrm{~m}, 1 \mathrm{H}), 5.69-5.74$ $(\mathrm{m}, 1 \mathrm{H}) ; \delta_{\mathrm{C}}\left(75 \mathrm{MHz}, \mathrm{CDCl}_{3}\right)-1.7,17.9,19.7,25.8,28.1,34.2,41.0,42.4$, $42.7,71.9,127.8,129.0 ; v($ film $) / \mathrm{cm}^{-1} 3023,2930,2855,1472,1253,1112$, 1013,835 .

1 For recent reviews of RCM see: R. H. Grubbs, S. J. Miller and G. C. Fu, Acc. Chem. Res., 1995, 28, 446; H.-G. Schmalz, Angew. Chem., Int. Ed. Engl., 1995, 34, 1833.

2 For selected examples see: S. F. Martin, Y. Liao, Y. Wong and T. Rein, Tetrahedron Lett., 1994, 35, 691; B. C. Borer, S. Deerenberg,
H. Beiraugel and U. K. Pandit, Tetrahedron Lett., 1994, 35, 3191 ; A. F. Houri, Z. Xu, D. A. Cogan and A. H. Hoveyda, J. Am. Chem. Soc., 1995, 117, 2943; J. S. Clark and J. G. Kettle, Tetrahedron Lett., 1997, 38, 123 and 127; A. Fürstner and K. Langemann, J. Org. Chem., 1996, 61, 8746; A. Kinoshita and M. Mori, J. Org. Chem. 1996, 61, 8356; K. C. Nicolaou, Y. He, D. Vourloumis, H. Valberg and Z. Yang, Angew. Chem., Int. Ed. Engl., 1996, 35, 2399.

3 M. D. E. Forbes, J. T. Patton, T. L. Myers, H. D. Maynard, D. W. Smith, Jr., G. R. Schulz and K. B. Wagener, J. Am. Chem. Soc., 1992, 114, 10978.

4 P. Schwab, R. H. Grubbs and J. W. Ziller, J. Am. Chem. Soc., 1996, 118, 100 .

5 R. R. Schrock, J. S. Murdzek, G. C. Bazan, J. Robbins, M. DiMare and M. O'Regan, J. Am. Chem. Soc., 1990, 112, 3875.

6 The molecular mechanics calculations were performed using the MM2 forcefield as implemented in the SPARTAN (ver. 4.1.1, Wavefunction, Inc., 18401 Von Karman Ave., \#370, Irvine, CA, 92715 USA, (C) 1995 Wavefunction, Inc.) and MACROMODEL (F. Mohamadi, N. G. J. Richards, W. C. Guida, R. Liskamp, M. Lipton, C. Caufield, G. Chang, T. Hendrickson and W. C. Still, J. Comput. Chem., 1990, 11, 440, ver. 3.5a, Dept. of Chemistry, Columbia University, NY, 10027) software packages.

7 R. J. Linderman, J. Siedlecki, S. A. O'Neill and H. Sun, J. Am. Chem. Soc., 1997, 119, 6919.

Received in Corvallis, OR, USA, 25th August 1997; 7/06200A 